\title{
A NOTE ON CARDINAL REFLECTIONS IN THE CATEGORY OF UNIFORM SPACES
}

\section{GIOVANNI VIDOSSICH}

In [5, Chapter iii, Exercise 2] it is shown that the countable uniform covers of any uniform space form a uniformity, and it is asked whether the same thing happens for arbitrary infinite cardinals. Here a partial affirmative answer is given: when its uniformity has a basis of $\sigma$-point-finite uniform covers ( a family of sets is said to be $\sigma$-pointfinite iff it is a union of countably many subfamilies each of which is a point-finite family), the space admits arbitrary cardinal reflections. This means that a positive solution of [5, Research Problem $\left.B_{3}\right]$ implies a positive solution of the above question. But the major interest of our result is that it furnishes the expected and most natural characterization of the uniformities defined in $[1, \S 3]$ by extended reticles: they are the cardinal reflections of the fine uniformity of the given space. From this, a very easy proof of the main results of [2] is derived, and it seems that almost all the work of G. Aquaro in that field may be considerably simplified at least in the technical apparatus (this is certainly true for [3]).

Our terminology and notations are based on [5] (but we do not assume $a$ priori the axiom $\mathrm{T}_{2}$ on uniform spaces).

1. Main results. Note that the uniformity $\nu$ of the following theorem is compatible with the topology of $X$ because it is finer than the precompact reflection $p \mu X$.

1.1 TheOREM. Let $\mu X$ be a uniform space and $\boldsymbol{\aleph}$ any infinite cardinal. If $\mu$ has a basis of $\sigma$-point-finite coverings, then the uniform covers of $\mu X$ with cardinality $\leqq \aleph$ form a uniformity $\nu$ on $X$.

Proof. We must prove that every uniform covering of $\mu X$ of

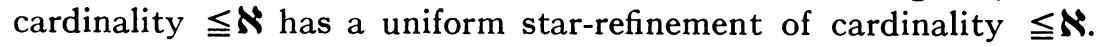
We begin by establishing the following result:

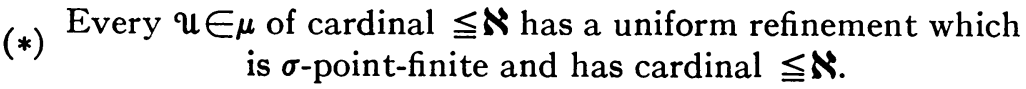

By hypothesis, there is a uniform refinement $\left(V_{n \alpha}\right)_{n \alpha}$ of $u$ such that, for each $n \in Z^{+},\left(V_{n \alpha}\right)_{\alpha \in A_{n}}$ is point-finite. Let $\leqq$ be a well-ordering for $\mathcal{U}$ and, for each $n \in Z^{+}$and each $U \in \mathcal{U}$, let $A_{U}^{n}=\left\{\alpha \in A_{n} \mid V_{n \alpha} \subseteq U\right\}$. Obviously the sets

Received by the editors March 3, 1969. 


$$
B_{U}^{n}=A_{U}^{n} \backslash \cup\left\{A_{U^{\prime}}^{n} \mid L^{\prime} \in \mathfrak{U}, L^{\prime \prime}<C^{\prime}\right\} \quad(U \in \mathfrak{u})
$$

form a partition of $A_{n}$. Define $W_{n U}=\bigcup\left\{V_{n \alpha} \mid \alpha \in B_{U}^{n}\right\}$. Then $\left(W_{n U}\right)_{n U}$ refines $\mathcal{U}$, is refined by $\left(V_{n \alpha}\right)_{n \alpha}$ and hence is a uniform cover of $\mu X$, and its index set $\boldsymbol{Z}^{+} \times \boldsymbol{u}$ has cardinality $\leqq \boldsymbol{N}$. Finally, for each $n \in \boldsymbol{Z}^{+}$, $\left(W_{n U}\right)_{U \in \mathcal{U}}$ is point-finite: to any $x \in X$ there corresponds a finite set $\left\{\alpha_{1}, \cdots, \alpha_{m}\right\} \subseteq A_{n}$ such that $\left\{V_{n \alpha_{1}}, \cdots, V_{n \alpha_{m}}\right\}$ is exactly the set of all $V_{n \alpha}$ 's which contain $x$. Then $x$ lies exactly in the members of the finite subfamily $\left(W_{n U_{1}}, \cdots, W_{n U_{m}}\right)$ of $\left(W_{n U}\right)_{U}$, where $U_{i}$ is defined by $\alpha_{i} \in B_{U_{i}}^{n}$. Thus $\left(^{*}\right)$ holds.

The following reasoning is largely inspired to the proof of [4,

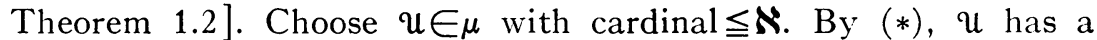
uniform refinement $\left(V_{n \alpha}\right)_{n \alpha}$ such that, for each $n \in \boldsymbol{Z}^{+},\left(V_{n \alpha}\right)_{\alpha \in A_{n}}$ is point-finite and $\operatorname{Card}\left(A_{n}\right) \leqq \boldsymbol{N}$. Let $J=\left(T_{\lambda}\right)_{\lambda} \in \mu$ be such that $\Im^{* *}$ refines $\left(V_{n \alpha}\right)_{n \alpha}$ and $T_{\lambda} \neq \varnothing$ for all $\lambda$. Define:

$$
\begin{aligned}
& m(\lambda)=\min \left\{n \in \boldsymbol{Z}^{+} \mid \operatorname{St}\left(T_{\lambda}, J\right) \subseteq V_{n \alpha} \text { for some } \alpha \in A_{n}\right\} ; \\
& n(\lambda)=\min \left\{n \in \boldsymbol{Z}^{+} \mid \operatorname{St}\left(\operatorname{St}\left(T_{\lambda}, \Im\right), \Im\right) \subseteq V_{n \alpha} \text { for some } \alpha \in A_{n}\right\} ; \\
& q(\lambda)=\left\{(n, \alpha) \mid n \leqq n(\lambda), V_{n \alpha} \supseteq T_{\lambda}\right\} .
\end{aligned}
$$

Because every $T_{\lambda}$ is nonempty and every $\left(V_{n \alpha}\right)_{\alpha}$ is point-finite, $q(\lambda)$ is a finite subset of $\boldsymbol{Z}^{+} \times \bigcup_{n=1}^{\infty} A_{n}$. For all $m, n \in \boldsymbol{Z}^{+}$and all finite subsets $F$ of $\boldsymbol{Z}^{+} \times \cup_{n=1}^{\infty} A_{n}$, define

$$
W_{m n F}=\bigcup\left\{T_{\lambda} \mid m(\lambda)=m, n(\lambda)=n, q(\lambda)=F\right\} .
$$

Clearly $\left(W_{m n F}\right)_{m n F}$ has cardinality $\leqq \aleph$ and is refined by $J$, so that it is a uniform cover of $\mu X$. For every $\lambda_{0}$ there is $\alpha\left(\lambda_{0}\right) \in A_{m\left(\lambda_{0}\right)}$ such that $\operatorname{St}\left(T_{\lambda_{0}}, \Im\right) \subseteq V_{m\left(\lambda_{0}\right) \alpha\left(\lambda_{0}\right)}$. If $W_{m n F} \cap W_{m\left(\lambda_{0}\right) n\left(\lambda_{0}\right) q\left(\lambda_{0}\right)} \neq \varnothing$, then there is a $\lambda$ such that $m(\lambda)=m, n(\lambda)=n, q(\lambda)=F$ and $T_{\lambda} \cap T_{\lambda_{0}} \neq \varnothing$. It follows that $T_{\lambda_{0}} \subseteq \operatorname{St}\left(T_{\lambda}, J\right)$ and $\operatorname{St}\left(T_{\lambda_{0}}, J\right) \subseteq \operatorname{St}\left(\operatorname{St}\left(T_{\lambda}, J\right), J\right) \subseteq V_{n(\lambda) \alpha(\lambda)}$ for a suitable $\alpha(\lambda) \in A_{n(\lambda)}$. Therefore $m\left(\lambda_{0}\right) \leqq n(\lambda)$. From this and $T_{\lambda} \subseteq \operatorname{St}\left(T_{\lambda_{0}}, J\right) \subseteq V_{m\left(\lambda_{0}\right) \alpha\left(\lambda_{0}\right)}$ it follows that $\left(m\left(\lambda_{0}\right), \alpha\left(\lambda_{0}\right)\right) \in q(\lambda)=F$, which implies $W_{m n F} \subseteq V_{m\left(\lambda_{0}\right) \alpha\left(\lambda_{0}\right)}$ : hence $\left(W_{m n F) m n F}\right.$ is a uniform starrefinement of $\mathcal{U}$. Q.E.D.

Now we desire to recall the definition-given in $[1, \S 3]$-of what G. Aquaro calls $\aleph$-uniformity of a given topological space $X, \boldsymbol{\aleph}$ being an infinite cardinal. Because the original definition is fairly complicated, we prefer to give an equivalent definition (the equivalence follows from $[1, \S 2$, Theorem 1-(e)]) which is more closely related to our purposes: The $\mathbf{\aleph}$-uniformity of $X$ has a base of uniform coverings consisting of all families $\left(U_{i}\right)_{i \in I}$ such that $\operatorname{Card}(I) \leqq \aleph,\left(U_{i}\right)_{i}$ is a locally finite open cover of $X$ and there is a closed covering $\left(F_{i}\right)_{i \in I}$ of $X$ and an entourage $W$ of a uniformity whose topology is less fine 
than the topology of $X$ for which $W\left[F_{i}\right] \subseteq U_{i}$ holds for all $i \in I$. Recall that the fine uniformity of a uniformizable space is the finest uniformity compatible with its topology.

The following result-which may be read alternatively: the $\boldsymbol{\aleph}_{\alpha^{-}}$ uniformity of $X$ is the $\boldsymbol{\aleph}_{\alpha+1}$-reflection of the fine uniformity of $X$ also implies the same conclusion when $X$ is not assumed uniformizable, as in Aquaro's works: it suffices to apply it to the weak topology determined by the set of all continuous mappings $X \rightarrow R$.

1.2. Corollary. For every uniformizable space $X$, the $\mathbf{\aleph}$-uniformity of $X$ is exactly the uniformity having as basis the set of all uniform coverings of the fine uniformity of $X$ which have cardinal $\leqq \aleph$.

Proof. Because the fine uniformity $\mu_{\infty}$ of $X$ has a basis of uniformly locally finite uniform coverings by $[\mathbf{5}$, vii.4] (or, alternatively, by the result of [1]), 1.1 implies that the set of all uniform coverings of $\mu_{\infty}$ having cardinal $\leqq \mathcal{N}$ is really the base for a uniformity $\mu$ on $X$ (this is the sole application of 1.1). Let $\nu$ be the $\boldsymbol{\aleph}$-uniformity of $X$. By the definition of $\nu$ given above (since $\{W[x] \mid x \in X\}$ are uniform covers whenever $W$ is an entourage of the given uniformity), it is clear that $\nu$ is less fine than $\mu$. Conversely, assume $\mathcal{U} \in \mu$ and $\operatorname{Card}(\mathcal{u}) \leqq \boldsymbol{N}$. Because $\mu_{\infty}$ has a base of locally finite uniform covers, we may repeat with obvious changes (it suffices to delete " $n$ " whenever it appears) the proof of statement $(*)$ in the proof of 1.1 to obtain a refinement $\mathcal{V}$ of $\mathcal{u}$ such that $V \in \mu_{\infty}, \operatorname{Card}(\mathcal{V}) \leqq \aleph$ and $V$ is an open locally finite cover of $X$. Let $\jmath \in \mu_{\infty}$ be an open star-refinement of $v$. For any $V \in \mathcal{V}$, define

$$
\Im_{V}=\{T \in \Im \mid \operatorname{St}(T, \Im) \subseteq V\} ; \quad Z_{V}=\bigcup\left\{T \mid T \in \Im_{V}\right\} .
$$

Since each element of $\Im$ is open, $\operatorname{St}\left(\bar{Z}_{V}, J\right) \subseteq U\left\{\operatorname{St}(T, J) \mid T \in J_{V}\right\} \subseteq V$. This implies the existence of an entourage $W$ of $\mu_{\infty} X$ such that $W\left[\bar{Z}_{V}\right] \subseteq V$ for all $V \in \mathcal{V}$. Thus $v \in \nu$. Q.E.D.

2. Applications to G. Aquaro's works. The aim of this section is to show how 1.2 may be used to simplify some proofs of [2]: the best result is (b), the simplification of the original proof of [2, Proposition 7] avoiding the use of the very complicated [2, Proposition 6]. Also the proofs of [3, Lemmas 2 and 3, Proposition 6] may be simplified, but we examine only [2] because the simplifications of [3] are not so spectacular.

Recall that, according to [2], a uniformizable space $X$ is said to be $a_{\boldsymbol{\aleph}}$-complete, with $\boldsymbol{\aleph}$ an infinite cardinal, iff the $\boldsymbol{\aleph}$-uniformity of $X$ is complete. 
(a) [2, Proposition 5]. Any product of $\boldsymbol{a}_{\mathbb{N}}$-complete spaces is $a_{\mathbb{N}}$-complete.

Proof. By [5, Chapter ii, Exercise 2], the product of uniformities

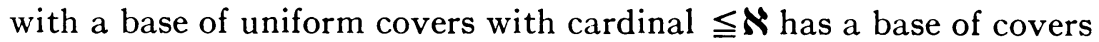
with cardinality $\leqq \boldsymbol{N}$. Then the conclusion follows from 1.2 and the fact that every uniformity finer than a complete one is complete. Q.E.D.

(b) [2, Proposition 7]. If $\mu$ is the $\aleph$-uniformity of $X$, then there is an $a_{\mathbb{N}}$-complete space $Y$ such that $\mu X$ is isomorphic to a dense subspace of $\nu Y, \nu$ being the $\boldsymbol{\aleph}$-uniformity of $Y$.

Proof. Let $Y$ be the completion of $\mu X$. By [5, ii.9], $\{\bar{U} \mid U \in \mathcal{u}\}$ is a uniform cover of $Y$ for all $u \in \mu$. By this and 1.2, the uniformity of $Y$ is less fine than $\nu$, the $\boldsymbol{N}$-uniformity of $Y$. Thus $\nu$ is complete and, again by 1.2 , induces on $X$ exactly $\mu$. Q.E.D.

\section{REFERENCES}

1. G. Aquaro, Ricovrimenti aperti e strutture uniformi sopra uno spazio topologico, Ann. Mat. Pura Appl. 47 (1959), 319-390.

2. ——, Completamenti di spazii uniformi, Ann. Mat. Pura Appl. 56 (1961), 87-98.

3. - Spazii collettivamente normali ed estensione di applicazioni continue, Riv. Mat. Univ. Parma 2 (1961), 77-90.

4. S. Ginsburg and J. R. Isbell, Some operators on uniform spaces, Trans. Amer. Math. Soc. 93 (1959), 145-168.

5. J. R. Isbell, Uniform spaces, Math. Surveys, No. 12, Amer. Math. Soc., Providence, R. I., 1964.

University of Pisa 